\title{
ON GLOBAL HÖLDER ESTIMATES FOR OPTIMAL TRANSPORTATION
}

\author{
ALEXANDER V. KOLESNIKOV
}

\begin{abstract}
We generalize a well-known result of L. Caffarelli on Lipschitz estimates for optimal transportation $T$ between uniformly log-concave probability measures. Let $T: \mathbb{R}^{d} \rightarrow \mathbb{R}^{d}$ be an optimal transportation pushing forward $\mu=e^{-V} d x$ to $\nu=e^{-W} d x$. Assume that 1) the second differential quotient of $V$ can be estimated from above by a power function, 2) modulus of convexity of $W$ can be estimated from below by $A_{q}|x|^{1+q}, q \geq 1$. Under these assumptions we show that $T$ is globally Hölder with a dimension-free coefficient. In addition, we study optimal transportation $T$ between $\mu$ and the uniform measure on a bounded convex set $K \subset \mathbb{R}^{d}$. We get estimates for the Lipschitz constant of $T$ in terms of $d$, $\operatorname{diam}(\mathrm{K})$ and $D V, D^{2} V$.
\end{abstract}

Keywords: optimal transportation, Monge-Ampère equation, Hölder apriori estimates, convex bodies, log-concave measures, isoperimetric and concentration inequalities

\section{INTRODUCTION}

According to a well-known result of L. Caffarelli [9] any optimal transportation mapping $T$ pushing forward the standard Gaussian measure $\gamma$ to a probability measure $e^{-W} \cdot \gamma$ with convex $W$ is 1-Lipschitz. This remarkable observation allows to recover many interesting results on analytic properties of uniformly convex measures. For instance, the Bakry-Emery condition for the log-Sobolev inequality ("flat" part) [1, Bakry-Ledoux comparison theorem [2, some correlation inequalities [9], 13]. Some recent generalizations of [9] can be found in 24].

The estimates of this type go back to A.V. Pogorelov [19] (see also "Pogorelov lemma" in 12]). According to his result, any the smooth solution of the MongeAmpère equation $\operatorname{det} D^{2} \varphi=1$ in a smooth domain $\Omega \subset \mathbb{R}^{d}$ satisfies

$$
C_{1}\left(\Omega, \Omega^{\prime}\right) \leq D^{2} \varphi(x) \leq C_{2}\left(\Omega, \Omega^{\prime}\right)
$$

$x \in \Omega^{\prime}$, where $\Omega^{\prime} \subseteq \Omega$ is another domain. The Hölder estimates play fundamental role in the regularity theory of partial differential equations. The overview of results on a priori estimates for fully nonlinear differential equations see in [15], [10]. For a more special case of the Monge-Ampère equation the readers are advised to consult [12], 23, 25].

On the other hand, mass transportation method is widely used in probability for establishing diverse isoperimetric and concentration properties. Given a "good" measure $\mu$ with known isoperimetric properties (e.g. Gaussian, product measures) and another measure $\nu$ one can try to transform $\mu$ into $\nu$ and deduce the desired information about $\nu$ from the properties of the mass transport (Lipschitz or Hölder estimates). Let us indicate some typical situations 
1) $\mu$ is Gaussian and $\nu$ is another product measure (see [21, [16])

2) $\nu$ is the uniform measure on a convex set and $\mu$ is log-concave with the same modulus of convexity (see, for instance, [5], 16, [17, and references therein)

3) $\mu$ is Gaussian and $D^{2} W \geq C$ (situation of the Caffarelli theorem).

The paper is organized as follows. In Section 2 we consider the optimal transportation $T$ pushing forward $e^{-V} d x$ to $e^{-W} d x$. Assume that $V$ and $W$ satisfy

$$
\begin{gathered}
V(x+y)+V(x-y)-2 V(x) \leq A_{p}|y|^{p+1}, \\
W(x+y)+W(x-y)-2 W(x) \geq A_{q}|y|^{q+1}
\end{gathered}
$$

with $0 \leq p \leq 1 \leq q$ and some positive $A_{p}, A_{q}$. We show that $T$ is globally $\frac{p+1}{q+1}$ Hölder:

$$
|T(x)-T(y)| \leq C|x-y|^{\frac{p+1}{q+1}},
$$

with $C$ depending only on $p, q, A_{p}, A_{q}$.

In particular, this implies the following. Let $\nu=e^{-W} d x$ be a probability measure satisfying

$$
W(x+y)+W(x-y)-2 W(x) \geq C_{\beta}|y|^{2 \beta}
$$

with $\beta \geq 1$. Then there exists $C$ depending only on $\beta, C_{\beta}$ such that $\nu$ satisfies the following concentration inequality:

$$
\nu\left(B_{r}\right) \geq 1-e^{-C|r|^{2 \beta}}
$$

for every $B \subset \mathbb{R}^{d}$ with $\nu(B) \geq \frac{1}{2}, B_{r}=\{y:|x-y| \leq r, x \in B\}$. This is a previously known consequence of a modified log-Sobolev inequality proved by Bobkov and Ledoux [5]. The Bobkov-Ledoux result has been generalized in [11] by transportation arguments. The corresponding isoperimetric inequalities have been proved in [17, by localization techniques.

In Sections 3-4 we prove estimates of the following type:

$$
\left\|D^{2} \varphi\right\| \leq f(\nabla \varphi),
$$

where $f$ is a non-negative function and $\nabla \varphi$ is the optimal mass transport pushing forward a smooth measure $\mu$ to $\nu=\left.\frac{1}{\lambda(K)} \cdot \lambda\right|_{K}$, where $K \subset \mathbb{R}^{d}$ is convex and $\lambda$ is the Lebesgue measure. In particular, we show that any uniform measure $\nu$ on a bounded convex set $K$ is a Lipschitz image of the standard Gaussian measure which Lipschitz constant does not exceed $C \sqrt{d} \cdot \operatorname{diam}(K)$ with some universal $C$.

After publishing online a preliminary version of this paper, the author obtained a remark from the participants of the Convex Analysis seminar in Tel-Aviv University. They noticed that the proof of Proposition 2.7 can be significantly simplified for the case of convex functions. The arguments are presented in Appendix. Consider a log-concave measure $\nu=e^{-W} d x$ such that

$$
W(x+y)+W(x-y)-W(x) \geq \delta(|y|)
$$

with a non-negative increasing function $\delta$. Then the optimal transportation of the standard Gaussian measure to $\nu$ satisfies the following:

$$
|\nabla \varphi(x)-\nabla \varphi(y)| \leq 8 \delta^{-1}\left(4|x-y|^{2}\right) .
$$

This implies, in particular, that $\nu$ admits the following dimension-free concentration property:

$$
\nu\left(B_{r}\right) \geq 1-\frac{1}{2} \exp _{2}\left(-\frac{1}{8} \delta(r / 8)\right)
$$


for every $B \subset \mathbb{R}^{d}$ with $\nu(B) \geq 1 / 2, B_{r}=\{y:|x-y| \leq r, x \in B\}$.

The author expresses his gratitude to the colleagues from the Tel-Aviv university, especially to Ronen Eldan and Sasha Sodin for communicating the proof of Lemma 5.1 and to Emanuel Milman for pointing out a mistake in the earlier version.

\section{Global Hölder estimates}

We deal throughout the paper with the standard finite-dimensional Euclidean space.

We recall that for every couple of probability measures $\mu_{1}=\rho_{1} d x, \mu_{2}=\rho_{2} d x$ there exists a mapping $T: \mathbb{R}^{d} \rightarrow \mathbb{R}^{d}$ pushing forward $\mu_{1}$ to $\mu_{2}$ which has the form $T=\nabla \varphi$, where $\varphi$ is a convex function (see [25] for details). For smooth $T$ one has the following change of variables formula (Monge-Ampère equation):

$$
\rho_{2}(\nabla \varphi) \operatorname{det} D^{2} \varphi=\rho_{1} .
$$

All the measures considered below are supposed to have a convex support of positive Lebegue measure.

Recall that a probability measure $\mu$ is called log-concave if it has the form $\mu=$ $e^{-V} d x$ with a convex $V$. Finally, recall that a mapping $T: \mathbb{R}^{d} \rightarrow \mathbb{R}^{d}$ is $M$-Lipschitz if

$$
\frac{|T(x)-T(y)|}{|x-y|} \leq M .
$$

By the Rademacher theorem $T$ is almost everywhere differentiable with $\|D T\| \leq M$. A mapping $T$ is $\alpha$-Hölder if there exists $0<\alpha \leq 1$ such that

$$
\frac{|T(x)-T(y)|}{|x-y|^{\alpha}} \leq M \text {. }
$$

We start with the one-dimensional case. Let $T=\varphi^{\prime}$ be the optimal transportation, pushing forward $\mu=e^{-V} d x$ to $\nu=e^{-W} d x$ with $\operatorname{supp}(\nu)=[a, b]$, where $V: \mathbb{R} \rightarrow \mathbb{R}$ and $W:(a, b) \rightarrow \mathbb{R}$ satisfy

$$
\begin{gathered}
\left|V^{\prime}(x)-V^{\prime}(y)\right| \leq C_{p}|x-y|^{p} \\
(x-y)\left(W^{\prime}(x)-W^{\prime}(y)\right) \geq C_{q}|x-y|^{q+1}
\end{gathered}
$$

for some $0<p \leq 1,1 \leq q$.

Measures $\mu$ and $\nu$ are supposed to be probability measures. We are looking for a maximum point $\left(t_{0}, x_{0}\right)$ of the following function

$$
F(t, x)=\frac{\varphi^{\prime}(x+t)-\varphi^{\prime}(x)}{|t|^{\alpha}} \operatorname{sign}(t), 0<\alpha \leq 1 .
$$

Remark 2.1. The arguments below are non-rigorous. A more general version of the result (with a rigorous proof) see in Corollary 2.9

Setting $t_{0}^{\prime}=-t_{0}$ and $x^{\prime}=x_{0}-t_{0}$ if necessary, we may assume that $t>0$. Differentiating in $x$ at the maximum point yields

Hence

$$
\begin{aligned}
\varphi^{\prime \prime}\left(x_{0}+t_{0}\right) & =\varphi^{\prime \prime}\left(x_{0}\right), \\
\varphi^{\prime \prime \prime}\left(x_{0}+t_{0}\right) & \leq \varphi^{\prime \prime \prime}\left(x_{0}\right) .
\end{aligned}
$$

$$
\frac{\varphi^{\prime \prime \prime}\left(x_{0}+t_{0}\right)}{\varphi^{\prime \prime}\left(x_{0}+t_{0}\right)}-\frac{\varphi^{\prime \prime \prime}\left(x_{0}\right)}{\varphi^{\prime \prime}\left(x_{0}\right)} \leq 0 .
$$


Taking derivative in $t$ we obtain

$$
\varphi^{\prime \prime}\left(x_{0}+t_{0}\right)=\frac{\alpha}{t_{0}}\left(\varphi^{\prime}\left(x_{0}+t_{0}\right)-\varphi^{\prime}\left(x_{0}\right)\right) .
$$

Let us differentiate the change of variables formula. One gets

$$
\frac{\varphi^{\prime \prime \prime}}{\varphi^{\prime \prime}}-W^{\prime}\left(\varphi^{\prime}\right) \varphi^{\prime \prime}=-V^{\prime}
$$

Using (3) we obtain

$$
\begin{aligned}
V^{\prime}\left(x_{0}+t_{0}\right)-V^{\prime}\left(x_{0}\right) & \geq\left(W^{\prime}\left(\varphi\left(x_{0}+t_{0}\right)\right)-W^{\prime}\left(\varphi^{\prime}\left(x_{0}\right)\right)\right) \varphi^{\prime \prime}\left(x_{0}+t\right) \\
& =\frac{\alpha}{t}\left[W^{\prime}\left(\varphi\left(x_{0}+t_{0}\right)\right)-W^{\prime}\left(\varphi^{\prime}\left(x_{0}\right)\right)\right]\left(\varphi^{\prime}\left(x_{0}+t_{0}\right)-\varphi^{\prime}\left(x_{0}\right)\right) .
\end{aligned}
$$

Then it follows from the assumptions on $V$ and $W$ that

$$
C_{p} t_{0}^{p+1} \geq \alpha C_{q}\left|\varphi^{\prime}\left(x_{0}+t_{0}\right)-\varphi^{\prime}\left(x_{0}\right)\right|^{q+1} .
$$

Hence

$$
\left(\frac{C_{p}}{\alpha C_{q}}\right)^{\frac{1}{q+1}} \geq\left|\varphi^{\prime}\left(x_{0}+t_{0}\right)-\varphi^{\prime}\left(x_{0}\right)\right| \cdot t_{0}^{-\frac{p+1}{q+1}} .
$$

Thus we get the following statement:

Theorem 2.2. Assume that $V: \mathbb{R} \rightarrow \mathbb{R}$ and $W: \mathbb{R} \rightarrow \mathbb{R}$ are continuously differentiable functions satisfying (11) and (2) for some $0 \leq p \leq 1, q \geq 1$. Set:

$$
0<\alpha:=\frac{p+1}{q+1} \leq 1 .
$$

Then

$$
\left|\varphi^{\prime}(x+t)-\varphi^{\prime}(x)\right| \leq\left(\frac{(q+1) C_{p}}{(p+1) C_{q}}\right)^{\frac{1}{q+1}}|t|^{\alpha} .
$$

Remark 2.3. A closed result has been established in 3]: any optimal mapping sending the one-dimensional exponential measure $\nu=\frac{1}{2} e^{-|x|} d x$ to $e^{-V} \cdot \nu$ with $\left|V^{\prime}\right| \leq 1-c, c>0$ is $\frac{1}{c}$-Lipschitz.

We assume that $V$ and $W$ satisfy

$$
\begin{gathered}
V(x+y)+V(x-y)-2 V(x) \leq A_{p}|y|^{p+1}, \\
W(x+y)+W(x-y)-2 W(x) \geq A_{q}|y|^{q+1},
\end{gathered}
$$

for some $0 \leq p \leq 1,1 \leq q, A_{p}>0, A_{q}>0$.

Remark 2.4. It can be easily verified that assumptions (4), (5) only make sense if $0 \leq p \leq 1,1 \leq q$. Indeed, assuming other values of $p, q$, one can easily deduce that the second derivatives of $W, V$ are either zero or infinity everywhere.

Theorem 2.5. Let $\mu$ and $\nu$ satisfy (4), (5) for some $0 \leq p \leq 1,1 \leq q$. Then $\varphi$ satisfies

$$
\varphi(x+t h)+\varphi(x-t h)-2 \varphi(x) \leq 2\left(\frac{A_{p}}{A_{q}}\right)^{\frac{1}{q+1}} t^{1+\alpha}
$$

for every unit vector $h \in \mathbb{R}^{d}$ with $\alpha=\frac{p+1}{q+1}$. 
Proof. To prove the multi-dimensional case we follow the arguments of Caffarelli from [9]. We consider the differential quotient

$$
\delta_{2} \varphi(x)=\varphi(x+t h)+\varphi(x-t h)-2 \varphi(x) \geq 0
$$

for some vector $h \in \mathbb{R}^{d}$ with $|h|=1$. Without loss of generality we may assume that the support of $\nu$ is a bounded convex domain. Note that according to a result from [7] $\varphi$ is twice continuously differentiable. It follows from the arguments of [9] that $\lim _{x \rightarrow \infty} \delta_{2} \varphi(x)=0$. Thus there exists a maximum point $x_{0}$ of $\delta_{2} \varphi(x)$. Differentiating at $x_{0}$ yields

$$
\begin{gathered}
\nabla \varphi\left(x_{0}+t h\right)+\nabla \varphi\left(x_{0}-t h\right)=2 \nabla \varphi\left(x_{0}\right), \\
D^{2} \varphi\left(x_{0}+t h\right)+D^{2} \varphi\left(x_{0}-t h\right) \leq 2 D^{2} \varphi\left(x_{0}\right) .
\end{gathered}
$$

It follows from the concavity of determinant that

$$
\begin{aligned}
\operatorname{det} D^{2} \varphi\left(x_{0}\right) & \geq \operatorname{det}\left(\frac{D^{2} \varphi\left(x_{0}+t h\right)+D^{2} \varphi\left(x_{0}-t h\right)}{2}\right) \\
& \geq\left(\operatorname{det} D^{2} \varphi\left(x_{0}+t h\right) \operatorname{det} D^{2} \varphi\left(x_{0}-t h\right)\right)^{\frac{1}{2}} .
\end{aligned}
$$

Applying the change of variables formula $\operatorname{det} D^{2} \varphi=e^{W(\nabla \varphi)-V}$ one finally gets

$$
\begin{array}{rl}
V\left(x_{0}+t h\right)+V\left(x_{0}-t h\right)-2 & V\left(x_{0}\right) \geq \\
W\left(\nabla \varphi\left(x_{0}+t h\right)\right)+W\left(\nabla \varphi\left(x_{0}-t h\right)\right)-2 W\left(\nabla \varphi\left(x_{0}\right)\right) .
\end{array}
$$

It follows from (7) that $v:=\nabla \varphi\left(x_{0}+t h\right)-\nabla \varphi\left(x_{0}\right)=\nabla \varphi\left(x_{0}\right)-\nabla \varphi\left(x_{0}-t h\right)$. Hence we get by (8) that

$A_{p} t^{p+1} \geq A_{q}\left|\nabla \varphi\left(x_{0}+t h\right)-\nabla \varphi\left(x_{0}\right)\right|^{q+1}=A_{q}\left|\nabla \varphi\left(x_{0}-t h\right)-\nabla \varphi\left(x_{0}\right)\right|^{q+1}=A_{q}|v|^{q+1}$.

By convexity of $\varphi$

$$
\begin{aligned}
\varphi\left(x_{0}+t h\right)+\varphi\left(x_{0}-t h\right)-2 \varphi\left(x_{0}\right) & \leq t\left\langle\nabla \varphi\left(x_{0}+t h\right)-\nabla \varphi\left(x_{0}-t h\right), h\right\rangle \\
& =2 t\langle v, h\rangle \leq 2 t|v| .
\end{aligned}
$$

Finally

$$
A_{p} t^{p+1} \geq A_{q}\left(\frac{\delta_{2} \varphi}{2 t}\right)^{q+1}
$$

Remark 2.6. For the case $p=q=1$ the estimate is known to be slightly better:

$$
\varphi(x+t h)+\varphi(x-t h)-2 \varphi(x) \leq\left(\frac{A_{p}}{A_{q}}\right)^{\frac{1}{2}} t^{2} .
$$

Our next goal is to establish Hölder continuity of $\nabla \varphi$.

Proposition 2.7. Let $u: \mathbb{R}^{d} \rightarrow \mathbb{R}$ be a differentiable function satisfying

$$
|u(x+y)+u(x-y)-2 u(x)| \leq C|y|^{\alpha+1},
$$

$\alpha>0$ and $u,|\nabla u|$ be integrable with respect to every Gaussian measure. Then there exists a constant $C^{\prime}$ depending only on $\alpha, C$ and $d$ such that

$$
\left|u_{v}(x+y)-u_{v}(x)\right| \leq C^{\prime}|y|^{\alpha}
$$

for every unit vector $v \in \mathbb{R}^{d}$ and every $x, y \in \mathbb{R}^{d}$. 
Proof. It is known that every bounded $f$ satisfying

$$
|f(x+y)+f(x-y)-2 f(x)| \leq C|y|^{\alpha+1}
$$

admits a Hölder continuous derivative: $|\nabla f(x+y)-\nabla f(x)| \leq A|y|^{\alpha}$ (see [20, Chapter 5(4), Proposition 9). Below we give a modification of the proof from 20.

Let us consider the heat semigroup acting on $u$.

$$
P_{t} u=\int_{\mathbb{R}^{d}} u(x-y) p_{t}(y) d y, \quad p_{t}(y)=\frac{1}{(2 \pi t)^{d / 2}} e^{-\frac{y^{2}}{2 t}} .
$$

We fix an orthonormal basis $\left\{e_{i}\right\}$. Clearly, it is sufficient to prove the statement for

$$
\left|u_{x_{i}}\left(x+h e_{j}\right)-u_{x_{i}}(x)\right|
$$

with any $1 \leq i, j \leq d$ and some fixed $h>0$. Note that $\left|u_{x_{i}}\left(x+h e_{j}\right)-u_{x_{i}}(x)\right|$ does not exceed

$\left|P_{t} u_{x_{i}}\left(x+h e_{j}\right)-P_{t} u_{x_{i}}(x)\right|+\left|u_{x_{i}}\left(x+h e_{j}\right)-P_{t} u_{x_{i}}\left(x+h e_{j}\right)\right|+\left|u_{x_{i}}(x)-P_{t} u_{x_{i}}(x)\right|$, for every $t>0$.

The first term is estimated by

$$
\begin{aligned}
P_{t} & u_{x_{i}}\left(x+h e_{j}\right)-P_{t} u_{x_{i}}(x)=\int_{0}^{h} \int_{\mathbb{R}^{d}} u_{x_{i} x_{j}}\left(x+r e_{j}-y\right) p_{t}(y) d y d r \\
& =\int_{0}^{h} \int_{\mathbb{R}^{d}} u\left(x+r e_{j}-y\right) p_{t}(y)_{y_{i} y_{j}} d y d r \\
& =\frac{1}{2} \int_{0}^{h} \int_{\mathbb{R}^{d}}\left(u\left(x+r e_{j}-y\right)+u\left(x+r e_{j}+y\right)-u\left(x+r e_{j}\right)\right) p_{t}(y)_{y_{i} y_{j}} d y d r \\
& \leq \frac{1}{2} \int_{0}^{h} \int_{\mathbb{R}^{d}}\left|u\left(x+r e_{j}-y\right)+u\left(x+r e_{j}+y\right)-u\left(x+r e_{j}\right)\right|\left|p_{t}(y)_{y_{i} y_{j}}\right| d y d r .
\end{aligned}
$$

Obviously

Hence

$$
p_{t}(y)_{y_{i} y_{j}}=\frac{1}{t}\left(\frac{y_{i} y_{j}}{t}-\delta_{i j}\right) p_{t}(y)
$$

$$
\left|P_{t} u_{x_{i}}\left(x+h e_{j}\right)-P_{t} u_{x_{i}}(x)\right| \leq \frac{C}{2 t} \int_{0}^{h} \int_{\mathbb{R}^{d}}|y|^{1+\alpha}\left|\frac{y_{i} y_{j}}{t}-\delta_{i j}\right| p_{t}(y) d y d r .
$$

It is clear by scaling arguments that the latter does not exceed

$$
C_{1}(C, \alpha, d) h \cdot t^{\frac{\alpha-1}{2}} \text {. }
$$

Thus we get for $t=h^{2}$ :

$$
\left|P_{t} u_{x_{i}}\left(x+h e_{j}\right)-P_{t} u_{x_{i}}(x)\right| \leq C_{1}(C, \alpha, d) h^{\alpha} .
$$

Let us estimate the remaining terms. To this end we consider $\left(P_{t} u\right)_{t}$. One gets

$$
\begin{aligned}
& \left(P_{t} u\right)_{t}(x)=\int_{\mathbb{R}^{d}} u(x-y)\left[\frac{1}{(2 \pi t)^{d / 2}} e^{-\frac{y^{2}}{2 t}}\right]_{t} d y \\
& =\int_{\mathbb{R}^{d}} u(x-y)\left(\frac{y^{2}}{2 t^{2}}-\frac{d}{2 t}\right) p_{t}(y) d y \\
& =\frac{1}{2} \int_{\mathbb{R}^{d}}(u(x-y)+u(x+y)-2 u(x))\left(\frac{y^{2}}{2 t^{2}}-\frac{d}{2 t}\right) p_{t}(y) d y .
\end{aligned}
$$


Hence

$$
\left|\left(P_{t} u\right)_{t}(x)\right| \leq \frac{1}{2} \int_{\mathbb{R}^{d}}|y|^{\alpha+1}\left|\frac{y^{2}}{2 t^{2}}-\frac{d}{2 t}\right| p_{t}(y) d y \leq C_{2}(C, \alpha, d) \cdot t^{\frac{\alpha-1}{2}} .
$$

Integration yields

$$
\left|P_{t} u(x)-u(x)\right| \leq C_{3}(C, \alpha, d) \cdot t^{\frac{\alpha+1}{2}}
$$

Note that

$$
\begin{gathered}
u_{x_{i}}(x)-P_{t} u_{x_{i}}(x)=-\int_{0}^{t}\left(P_{s} u\right)_{s x_{i}} d s, \\
P_{s} u=P_{s / 2} u * p_{s / 2} .
\end{gathered}
$$

Differentiating the convolution identity yields

$$
\begin{aligned}
P_{t} u_{x_{i}}(x)-u_{x_{i}}(x) & =\int_{0}^{t}\left(\int_{\mathbb{R}^{d}}\left(P_{s / 2} u(y)\right)_{s}\left(p_{s / 2}(x-y)\right)_{x_{i}} d y\right) d s \\
& +\int_{0}^{t}\left(\int_{\mathbb{R}^{d}} P_{s / 2} u(y)\left(p_{s / 2}(x-y)\right)_{x_{i} s} d y\right) d s
\end{aligned}
$$

It is easy to check that

$$
\begin{aligned}
& \int_{0}^{t}\left(\int_{\mathbb{R}^{d}} P_{s / 2} u(y)\left(p_{s / 2}(x-y)\right)_{x_{i} s} d y\right) d s= \\
& =\int_{0}^{t}\left(\int_{\mathbb{R}^{d}}\left(P_{s / 2} u-u\right)(y)\left(p_{s / 2}(x-y)\right)_{x_{i} s} d y\right) d s+P_{t / 2} u_{x_{i}}(x)-u_{x_{i}}(x) .
\end{aligned}
$$

One has for $t=h^{2}$

$$
\begin{aligned}
P_{t} u_{x_{i}}(x)-P_{t / 2} u_{x_{i}}(x) & =\int_{0}^{t}\left(\int_{\mathbb{R}^{d}}\left(P_{s / 2} u\right)_{s}(y)\left(p_{s / 2}(x-y)\right)_{x_{i}} d y\right) d s \\
& +\int_{0}^{t}\left(\int_{\mathbb{R}^{d}}\left(P_{s / 2} u-u\right)(y)\left(p_{s / 2}(x-y)\right)_{x_{i} s} d y\right) d s .
\end{aligned}
$$

Then it follows from (10), (9) that

$$
\begin{aligned}
& \left|P_{t} u_{x_{i}}(x)-P_{t / 2} u_{x_{i}}(x)\right| \leq \\
& \left.\left.C_{4}\left(\int_{0}^{t} s^{\frac{\alpha-1}{2}} \int_{\mathbb{R}^{d}} \mid p_{s / 2}(x-y)\right)_{x_{i}}\left|d y d s+\int_{0}^{t} s^{\frac{\alpha+1}{2}} \int_{\mathbb{R}^{d}}\right| p_{s / 2}(x-y)\right)_{x_{i} s} \mid d y d s\right) .
\end{aligned}
$$

Applying the same arguments as above we obtain

$$
\left|P_{t} u_{x_{i}}(x)-P_{t / 2} u_{x_{i}}(x)\right| \leq C_{5} \int_{0}^{t} s^{\frac{\alpha-2}{2}} d s=C_{6} t^{\frac{\alpha}{2}} .
$$

Hence

$$
\begin{aligned}
& \left|P_{t} u_{x_{i}}(x)-u_{x_{i}}(x)\right| \\
& \leq \sum_{k=0}^{\infty}\left|P_{t / 2^{k}} u_{x_{i}}(x)-P_{t / 2^{k+1}} u_{x_{i}}(x)\right| \leq C_{6} \sum_{k=0}^{\infty}\left(\frac{t}{2^{k}}\right)^{\frac{\alpha}{2}}=C_{7} t^{\alpha / 2}=C_{7} h^{\alpha} .
\end{aligned}
$$

The same estimate holds for the remaining third term. The proof is complete.

Remark 2.8. The statement does not hold for $\alpha=0$ (see [20]4.3.1). 
Corollary 2.9. Under assumptions of Theorem 2.5 the optimal transportation $\nabla \varphi$ is Hölder continuous:

$$
|\nabla \varphi(x)-\nabla \varphi(y)| \leq C|x-y|^{\alpha}
$$

with $C$ depending only on $p, q, A_{p}, A_{q}$.

Proof. Replace $\nu$ by $\left.\frac{1}{\nu(A)} \nu\right|_{A}$ with a bounded convex $A$. Clearly, the new potential satisfies assumptions of the theorem. Hence, using standard approximation arguments we can restrict ourselves to the case of compactly supported $\nu$. Thus we assume without loss of generality that $|\nabla \varphi| \leq K$. By Theorem 2.5

$$
\varphi(x+t h)+\varphi(x-t h)-2 \varphi(x) \leq C|t|^{\alpha}
$$

for every unit vector $h$. We get immediately from Proposition 2.7 that $\nabla \varphi$ is Hölder. To see that the constant does not depend on dimension, let as fix $x, h \in \mathbb{R}^{d}$ and $v \in \mathbb{R}^{d}$ with $|h|=|v|=1$. Consider the restriction of $\varphi$ onto the affine hyperplane $A_{x, v, h}=\{x+t h+s v, s \in \mathbb{R}, t \in \mathbb{R}\}$. This restriction clearly satisfies (11). Thus the 2-dimensional version of Lemma 2.7 implies that

$$
|\langle\nabla \varphi(x+t h)-\nabla \varphi(x), v\rangle|=\left|\varphi_{v}(x+t h)-\varphi_{v}(x)\right| \leq C^{\prime}|t|^{\alpha},
$$

where $C^{\prime}$ (a corresponding "two-dimensional" constant) does not depend on $d$ and on directions of $h, v$. Since this can be repeated for every $x, h, v$ with the same constant $C^{\prime}$, one has

$$
|\nabla \varphi(x+t h)-\nabla \varphi(x)|=\sup _{|v|=1}|\langle\nabla \varphi(x+t h)-\nabla \varphi(x), v\rangle| \leq C^{\prime}|t|^{\alpha} .
$$

The proof is complete.

Remark 2.10. A more general statement see in Corolary 5.2 .

\section{Estimates For the SECOND ORdeR DERIVATIVES: ONE-Dimensional CASE}

We start with some heuristic estimates in the one-dimensional case. Consider a convex function $\varphi$ such that $\varphi^{\prime}$ sends $\mu=\frac{1}{\sqrt{2 \pi}} e^{-\frac{x^{2}}{2}} d x$ to a probability measure $e^{-W} d x$. We assume that $W$ is sufficiently smooth.

By the change of variables formula

$$
-\frac{\log 2 \pi}{2}-\frac{x^{2}}{2}=-W\left(\varphi^{\prime}\right)+\log \varphi^{\prime \prime}
$$

Assume that $f$ is a smooth function such that $\frac{\varphi^{\prime \prime}}{f\left(\varphi^{\prime}\right)}$ admits its maximum at some point $x_{0}$. One has at this point

$$
\frac{\varphi^{(3)}}{f\left(\varphi^{\prime}\right)}-\frac{\left(\varphi^{\prime \prime}\right)^{2} f^{\prime}\left(\varphi^{\prime}\right)}{f^{2}\left(\varphi^{\prime}\right)}=0, \quad \frac{\varphi^{(4)}}{f\left(\varphi^{\prime}\right)}-\frac{3 \varphi^{(3)} \varphi^{\prime \prime} f^{\prime}\left(\varphi^{\prime}\right)}{f^{2}\left(\varphi^{\prime}\right)}-\left(\varphi^{\prime \prime}\right)^{3}\left[\frac{f^{\prime}}{f^{2}}\right]^{\prime}\left(\varphi^{\prime}\right) \leq 0 .
$$

Thus we get

$$
\varphi^{(3)}=\frac{\left(\varphi^{\prime \prime}\right)^{2} f^{\prime}\left(\varphi^{\prime}\right)}{f\left(\varphi^{\prime}\right)}
$$

Applying (14) we find

$$
\varphi^{(4)} \leq\left(\varphi^{\prime \prime}\right)^{3}\left[\frac{f^{\prime \prime}}{f}+\left(\frac{f^{\prime}}{f}\right)^{2}\right]\left(\varphi^{\prime}\right) .
$$


Differentiating (12) twice yields

$$
0=1-W^{\prime \prime}\left(\varphi^{\prime}\right)\left(\varphi^{\prime \prime}\right)^{2}-W^{\prime}\left(\varphi^{\prime}\right) \varphi^{(3)}+\frac{\varphi^{(4)}}{\varphi^{\prime \prime}}-\left(\frac{\varphi^{(3)}}{\varphi^{\prime \prime}}\right)^{2} .
$$

Applying (14) and (15) we get

$$
-\left(\varphi^{\prime \prime}\right)^{2} \frac{f^{\prime \prime}\left(\varphi^{\prime}\right)}{f\left(\varphi^{\prime}\right)}+W^{\prime \prime}\left(\varphi^{\prime}\right)\left(\varphi^{\prime \prime}\right)^{2}+W^{\prime}\left(\varphi^{\prime}\right) \frac{\left(\varphi^{\prime \prime}\right)^{2} f^{\prime}\left(\varphi^{\prime}\right)}{f\left(\varphi^{\prime}\right)} \leq 1 .
$$

Now assume that $f$ satisfies $-\frac{f^{\prime \prime}}{f}+W^{\prime \prime}+W^{\prime} \frac{f^{\prime}}{f} \geq \frac{1}{f^{2}}$. Thus we get at $x_{0}$

$$
\left[\frac{\varphi^{\prime \prime}}{f\left(\varphi^{\prime}\right)}\right]^{2} \leq 1 \text {. }
$$

Since $x_{0}$ is supposed to be the point of maximum for $\frac{\varphi^{\prime \prime}}{f\left(\varphi^{\prime}\right)}$, we get a formal proof of the following statement:

Assume that $f$ and $W$ satisfy

$$
-\frac{f^{\prime \prime}}{f}+W^{\prime \prime}+W^{\prime} \frac{f^{\prime}}{f} \geq \frac{1}{f^{2}} .
$$

Then

$$
\varphi^{\prime \prime} \leq f\left(\varphi^{\prime}\right)
$$

Of course, these arguments are non-rigorous. Nevertheless, they are applicable for many reasonable situations.

Example 3.1. For the case $W^{\prime \prime} \geq K$ and $f \equiv \frac{1}{\sqrt{K}}$ we get a refinement of the Caffarelli's result:

$$
\varphi^{\prime \prime} \leq \frac{1}{\sqrt{K}}
$$

Example 3.2. Assume that

$$
e^{-W}=\frac{1}{2 a} I_{[-a, a]} .
$$

Applying the arguments from above, we get the following estimate:

$$
\varphi^{\prime \prime} \leq f\left(\varphi^{\prime}\right),
$$

where $f:[-a, a] \rightarrow \mathbb{R}^{+}$is a concave even function, decreasing of $[0, a]$, such that $f(a)=f(-a)=0$ and

$$
-f f^{\prime \prime}=1 .
$$

This function can be found explicitly:

$$
f(t)=f(0) \cdot \Phi^{-1}\left(\frac{\sqrt{\frac{\pi}{2}} f(0)-t}{f(0)}\right) .
$$

Here $\Phi:[0,1] \rightarrow\left[0, \sqrt{\frac{\pi}{2}}\right]$ is defined by

$$
\Phi(x)=\int_{0}^{x} \frac{d s}{\sqrt{-2 \ln s}}=\int_{\sqrt{-2 \ln x}}^{\infty} e^{-\frac{s^{2}}{2}} d s .
$$

In addition, $a$ and $f(0)$ are related by

$$
f(0)=a \sqrt{\frac{2}{\pi}} .
$$


Thus

$$
\varphi^{\prime \prime} \leq a \sqrt{\frac{2}{\pi}} \Phi^{-1}\left(\sqrt{\frac{\pi}{2}} \cdot \frac{a-\varphi^{\prime}}{a}\right) .
$$

Note that this example can be obtained directly from the Caffarelli theorem. Indeed, set:

$$
G(x)=\int_{0}^{x} \frac{d s}{f(s)}=-f^{\prime}(x)
$$

It can be verified by the direct computation that $G$ pushes forward $\frac{1}{2 a} I_{[-a, a]}$ to $\nu=\left.\frac{1}{\gamma([-b, b])} \cdot \gamma\right|_{[-b, b]}$, where $b=G(a)$. Note that $T=G\left(\varphi^{\prime}\right)$ is the optimal transportation of $\gamma$ to $\nu$. Hence, by the Caffarelli theorem, $T$ is 1-Lipschitz: $T^{\prime} \leq 1$. Thus $\frac{\varphi^{\prime \prime}}{f\left(\varphi^{\prime}\right)} \leq 1$.

\section{Estimates FOR THE SECOND ORDER DERIVATIVES: MULTIDIMENSIONAL CASE}

For the rest of the paper $\nu$ is the uniform measure on a bounded convex domain $K$. The potential $V$ is supposed to be at least two times differentiable with a Hölder second derivative.

Note that the assumption of convexity of $K$ is crucial for smoothness of $\varphi$. It is well known that for non-convex sets the potential $\varphi$ is not smooth in general. Nevertheless, in our case the Caffarelli's regularity theory ensures that $\varphi$ is smooth (see [7], 8]). Indeed, let us take any convex domain $K^{\prime} \subset K$ such that $\partial K^{\prime}$ lies positive distance from $K$. Let $K_{0}=T^{-1} K^{\prime}$. It is easy to check that $K_{0}$ is bounded (otherwise we get the contradiction with the monotonicity of $T$ ). Then applying a result from [7] we immediately get that $T$ is differentiable with a Hölder continuous derivative inside of $K_{0}$. The further regularity follows from the smoothness of $V$ by the classical arguments (see [7, Remark 4.15 in [25]).

Definition 4.1. For a positive $a$ and $p \in \mathbb{R}$ let $f(t):=f_{p, a}(t):[-a, a] \rightarrow \mathbb{R}^{+}$be a function satisfying:

1) $f f^{\prime \prime}+p\left(f^{\prime}\right)^{2}=-1$

2) $f$ is even and $f(a)=0$.

Assumptions 1)-2) define uniquely a function $f$, which decreases on $[0, a]$. Assume for a while that $p>0$. First, taking $f$ as a new variable and integrating 1 ), one gets

$$
f^{\prime}=-\frac{1}{\sqrt{p}} \sqrt{\left(\frac{f(0)}{f}\right)^{2 p}-1} .
$$

This yields the following expression for $f$ :

$$
f(t)=f(0) \sqrt[p]{\Psi^{-1}\left(\sqrt{p} \frac{t}{f(0)}\right)},
$$

where $\Psi:[0,1] \rightarrow\left[0, \int_{0}^{\frac{\pi}{2}} \sin ^{\frac{1}{p}} x d x\right]$

$$
\Psi(t)=\int_{t}^{1} \frac{r^{\frac{1}{p}} d r}{\sqrt{1-r^{2}}}
$$

In addition, $a$ and $f(0)$ are related by

$$
a \sqrt{p}=f(0) \int_{0}^{1} \frac{r^{\frac{1}{p}} d r}{\sqrt{1-r^{2}}}=f(0) \int_{0}^{\frac{\pi}{2}} \sin ^{\frac{1}{p}} x d x .
$$


The case $p=0$ has been considered in Example 3.2. The case $p<0$ is similar to $p>0$. One has

$$
f(t)=f(0) \sqrt[p]{\Psi^{-1}\left(\sqrt{-p} \frac{t}{f(0)}\right)},
$$

where $\Psi:[1, \infty] \rightarrow\left[0, \int_{0}^{\frac{\pi}{2}} \sin ^{-1-\frac{1}{p}} x d x\right]$

$$
\Psi(t)=\int_{1}^{t} \frac{r^{\frac{1}{p}} d r}{\sqrt{r^{2}-1}} .
$$

In addition, $a$ and $f(0)$ are related by

$$
a \sqrt{-p}=f(0) \int_{1}^{\infty} \frac{r^{\frac{1}{p}} d r}{\sqrt{r^{2}-1}}=f(0) \int_{0}^{\frac{\pi}{2}} \sin ^{-1-\frac{1}{p}} x d x
$$

Theorem 4.2. Let $K \subset \mathbb{R}^{d}, d>1$ be a bounded convex set of a positive volume. Let $\nabla \varphi: \mathbb{R}^{d} \rightarrow K$ be the optimal transportation pushing forward probability measure $\mu=e^{-V} d x$ with smooth $V$ to the uniform measure $\left.\frac{1}{\lambda(K)} \lambda\right|_{K}$. Assume that $V_{h h} \leq \Lambda$ for some $h \in \mathbb{R}^{d}$ with $|h|=1$. Then one has

$$
\varphi_{h h} \leq \sqrt{\Lambda} \cdot f_{\frac{d-1}{4}, a}\left(\varphi_{h}-t_{0}\right)
$$

where $t_{0}$ is chosen in such a way that

$$
L_{1}=\left\{x:\langle x, h\rangle=t_{0}-a\right\}, L_{2}=\left\{x:\langle x, h\rangle=t_{0}+a\right\}
$$

are supporting hyperplanes to $K$.

If, in addition, we assume that $\left|V_{h}\right| \leq M$ then the following dimension-free estimate holds:

for any $p>-1$.

$$
\varphi_{h h} \leq \sqrt{\Lambda+\frac{M^{2}}{4(1+p)}} \cdot f_{p, a}\left(\varphi_{h}-t_{0}\right)
$$

Proof. Note that the estimates are invariant with respect to any shift of the space. Let us shift $K$ is such a way that $K$ contains the origin. This clearly implies that $\lim _{x \rightarrow \infty} \varphi(x)=+\infty$. We are looking for a maximum of $\varphi_{h h}(x) e^{\psi\left(\varphi_{h}(x)\right)}$ among all of $x \in \mathbb{R}^{d}$, with $\psi$ to be chosen later. To apply the maximum principle and make sure that the maximum is attained we deal with the following compactly supported modification:

$$
F_{\varepsilon}(x)=(1-\varepsilon \varphi)+\varphi_{h h}(x) e^{\psi\left(\varphi_{h}(x)\right)} .
$$

Consider the change of variables formula for $\varphi$

$$
C(K)+\log \operatorname{det} D^{2} \varphi=-V
$$

Let $y$ be a desired maximum point of $F_{\varepsilon}$. All the computations below are made at this point. First we change the coordinate system linearly in such a way that

$$
\text { 1) } \left.h=e_{1}, 2\right) D^{2} \varphi(y) \text { is diagonal. }
$$

The first requirement is achieved just by a rotation. In addition, without loss of generality we may assume that $\left(D^{2} \varphi\right)_{i, j}, i, j \geq 2$ is diagonal. To fulfill the second one we choose a non-orthogonal linear transformation.

$$
x_{1}^{\prime}=x_{1}+\frac{\varphi_{x_{2} x_{1}}(y)}{\varphi_{x_{1} x_{1}}(y)} x_{2}+\cdots+\frac{\varphi_{x_{d} x_{1}}(y)}{\varphi_{x_{1} x_{1}}(y)} x_{d},
$$




$$
x_{i}^{\prime}=x_{i}, i \in\{2, \cdots, d\} .
$$

Here $\left(x_{1}^{\prime}, \cdots, x_{d}^{\prime}\right)$ is the "new" coordinate system and $\left(x_{1}, \cdots, x_{d}\right)$ is the "old" one. One checks easily that $\varphi_{x_{1}^{\prime} x_{i}^{\prime}}=0$ for $i \geq 2$. Since the transformation is represented by a unimodal matrix, the change of variables formula looks in new coordinates as follows:

(16) $C(K)+\log \operatorname{det} D_{x^{\prime}}^{2} \varphi=-V\left(x_{1}^{\prime}-\frac{\varphi_{x_{2} x_{1}}(y)}{\varphi_{x_{1} x_{1}}(y)} x_{2}^{\prime}-\cdots-\frac{\varphi_{x_{d} x_{1}}(y)}{\varphi_{x_{1} x_{1}}(y)} x_{d}^{\prime}, x_{2}^{\prime}, \cdots, x_{d}^{\prime}\right)$,

where $D_{x^{\prime}}^{2} \varphi$ denotes the matrix of second derivatives computed in new coordinate system. In what follows we set: $x_{1}^{\prime}-\frac{\varphi_{x_{2} x_{1}}(y)}{\varphi_{x_{1} x_{1}}(y)} x_{2}^{\prime}-\cdots-\frac{\varphi_{x_{d} x_{1}}(y)}{\varphi_{x_{1} x_{1}}(y)} x_{d}^{\prime}=O x^{\prime}$ and write for simplicity $x_{i}$ instead of $x_{i}^{\prime}$.

Let us differentiate ate (16). To this end it is convenient to use the following expression for the determinant

$$
\operatorname{det} D^{2} \varphi=\sum_{\sigma \in S(d)} \operatorname{sign}(\sigma) \varphi_{x_{1} x_{\sigma(1)}} \cdots \varphi_{x_{d} x_{\sigma(d)}}
$$

and taking into account that $\varphi_{x_{i} x_{j}}(y)=0$ if $i \neq j$, we get at $y$ :

$$
\begin{gathered}
-V_{x_{1}}(O x)=\frac{\left(\operatorname{det} D^{2} \varphi\right)_{x_{1}}}{\operatorname{det} D^{2} \varphi}=\sum_{i} \frac{\varphi_{x_{i} x_{i} x_{1}}}{\varphi_{x_{i} x_{i}}}, \\
-V_{x_{1} x_{1}}(O x)=\frac{\left(\operatorname{det} D^{2} \varphi\right)_{x_{1} x_{1}}}{\operatorname{det} D^{2} \varphi}-\left(\frac{\left(\operatorname{det} D^{2} \varphi\right)_{x_{1}}}{\operatorname{det} D^{2} \varphi}\right)^{2}= \\
=\sum_{i} \frac{\varphi_{x_{i} x_{i} x_{1} x_{1}}}{\varphi_{x_{i} x_{i}}}+\sum_{i \neq j} \frac{\varphi_{x_{i} x_{i} x_{1}}}{\varphi_{x_{i} x_{i}}} \frac{\varphi_{x_{j} x_{j} x_{1}}}{\varphi_{x_{j} x_{j}}}-\sum_{i \neq j} \frac{\varphi_{x_{i} x_{j} x_{1}}^{2}}{\varphi_{x_{i} x_{i}} \varphi_{x_{j} x_{j}}} .
\end{gathered}
$$

Differentiating (16) twice in $x_{1}$ yields

$$
-\Lambda \leq-V_{x_{1} x_{1}}(O x)=\left[\log \operatorname{det} D^{2} \varphi\right]_{x_{1} x_{1}}=\frac{\left(\operatorname{det} D^{2} \varphi\right)_{x_{1} x_{1}}}{\operatorname{det} D^{2} \varphi}-\left(\frac{\left(\operatorname{det} D^{2} \varphi\right)_{x_{1}}}{\operatorname{det} D^{2} \varphi}\right)^{2} .
$$

It follows from (17), (18) that

$$
-\Lambda \leq \sum_{i} \frac{\varphi_{x_{i} x_{i} x_{1} x_{1}}}{\varphi_{x_{i} x_{i}}}-\sum_{i, j} \frac{\varphi_{x_{i} x_{j} x_{1}}^{2}}{\varphi_{x_{i} x_{i}} \varphi_{x_{j} x_{j}}} .
$$

Since $\log F_{\varepsilon}$ admits its maximum at $y$, one has at this point

$$
\begin{gathered}
-\frac{\varepsilon}{1-\varepsilon \varphi} \varphi_{x_{1}}+\frac{\varphi_{x_{1} x_{1} x_{1}}}{\varphi_{x_{1} x_{1}}}+\psi^{\prime}\left(\varphi_{x_{1}}\right) \varphi_{x_{1} x_{1}}=0, \\
-\frac{\varepsilon}{1-\varepsilon \varphi} \varphi_{x_{i}}+\frac{\varphi_{x_{1} x_{1} x_{i}}}{\varphi_{x_{1} x_{1}}}=0, i \geq 1 .
\end{gathered}
$$

Differentiating $\log F_{\varepsilon}$ twice in $x_{1}$ yields

$$
\begin{aligned}
-\frac{\varepsilon}{1-\varepsilon \varphi} \varphi_{x_{i} x_{i}} & -\frac{\varepsilon^{2}}{(1-\varepsilon \varphi)^{2}} \varphi_{x_{i}}^{2}+\frac{\varphi_{x_{1} x_{1} x_{i} x_{i}}}{\varphi_{x_{1} x_{1}}}-\left(\frac{\varphi_{x_{1} x_{1} x_{i}}}{\varphi_{x_{1} x_{1}}}\right)^{2} \\
& +\psi^{\prime \prime}\left(\varphi_{x_{1}}\right) \varphi_{x_{1} x_{i}}^{2}+\psi^{\prime}\left(\varphi_{x_{1}}\right) \varphi_{x_{1} x_{i} x_{i}} \leq 0 .
\end{aligned}
$$


Multiplying this inequality on $\frac{\varphi_{x_{1} x_{1}}}{\varphi_{x_{i} x_{i}}}$, summing in $i$ and applying (19) one gets

$$
\begin{aligned}
& -\frac{d \varepsilon}{1-\varepsilon \varphi} \varphi_{x_{1} x_{1}}-\frac{\varepsilon^{2}}{(1-\varepsilon \varphi)^{2}} \varphi_{x_{1} x_{1}}\left(\frac{\varphi_{x_{1}}^{2}}{\varphi_{x_{1} x_{1}}}+\cdots+\frac{\varphi_{x_{d}}^{2}}{\varphi_{x_{d} x_{d}}}\right) \\
& +\sum_{i, j} \frac{\varphi_{x_{i} x_{j} x_{1}}^{2}}{\varphi_{x_{i} x_{i}} \varphi_{x_{j} x_{j}}}-\sum_{i} \frac{\varphi_{x_{1} x_{1} x_{i}}^{2}}{\varphi_{x_{1} x_{1}} \varphi_{x_{i} x_{i}}} \\
& +\psi^{\prime \prime}\left(\varphi_{x_{1}}\right) \varphi_{x_{1} x_{1}}^{2}+\psi^{\prime}\left(\varphi_{x_{1}}\right)\left[\sum_{i} \frac{\varphi_{x_{1} x_{i} x_{i}}}{\varphi_{x_{i} x_{i}}}\right] \varphi_{x_{1} x_{1}} \leq \Lambda .
\end{aligned}
$$

Note that

$$
\begin{aligned}
& \sum_{i, j} \frac{\varphi_{x_{i} x_{j} x_{1}}^{2}}{\varphi_{x_{i} x_{i}} \varphi_{x_{j} x_{j}}}-\sum_{i} \frac{\varphi_{x_{1} x_{1} x_{i}}^{2}}{\varphi_{x_{1} x_{1} \varphi_{x_{i} x_{i}}}}=\sum_{i \in\{2, \cdots, d\}} \sum_{j \in\{1, \cdots, d\}} \frac{\varphi_{x_{i} x_{j} x_{1}}^{2}}{\varphi_{x_{i} x_{i}} \varphi_{x_{j} x_{j}}} \\
& \geq \sum_{j \in\{2, \cdots, d\}} \frac{\varphi_{x_{j} x_{j} x_{1}}^{2}}{\varphi_{x_{j} x_{j}}^{2}}+\sum_{i \in\{2, \cdots, d\}} \frac{\varphi_{x_{i} x_{1} x_{1}}^{2}}{\varphi_{x_{i} x_{i}} \varphi_{x_{1} x_{1}}} .
\end{aligned}
$$

One gets by (21)

$$
\sum_{i, j} \frac{\varphi_{x_{i} x_{j} x_{1}}^{2}}{\varphi_{x_{i} x_{i}} \varphi_{x_{j} x_{j}}}-\sum_{i} \frac{\varphi_{x_{1} x_{1} x_{i}}^{2}}{\varphi_{x_{1} x_{1}} \varphi_{x_{i} x_{i}}} \geq \sum_{j \in\{2, \cdots, d\}} \frac{\varphi_{x_{j} x_{j} x_{1}}^{2}}{\varphi_{x_{j} x_{j}}^{2}}+\sum_{i \in\{2, \cdots, d\}} \frac{\varepsilon^{2}}{(1-\varepsilon \varphi)^{2}} \frac{\varphi_{x_{1} x_{1}}}{\varphi_{x_{i} x_{i}}} \varphi_{x_{i}}^{2} .
$$

Hence it follows from (22)

$$
\begin{aligned}
& -\frac{d \varepsilon}{1-\varepsilon \varphi} \varphi_{x_{1} x_{1}}-\frac{\varepsilon^{2}}{(1-\varepsilon \varphi)^{2}} \varphi_{x_{1}}^{2}+\sum_{j \in\{2, \cdots, d\}} \frac{\varphi_{x_{j} x_{j} x_{1}}^{2}}{\varphi_{x_{j} x_{j}}^{2}} \\
& +\psi^{\prime \prime}\left(\varphi_{x_{1}}\right) \varphi_{x_{1} x_{1}}^{2}+\psi^{\prime}\left(\varphi_{x_{1}}\right)\left[\sum_{i \in\{2, \cdots d\}} \frac{\varphi_{x_{1} x_{i} x_{i}}}{\varphi_{x_{i} x_{i}}}\right] \varphi_{x_{1} x_{1}}+\psi^{\prime}\left(\varphi_{x_{1}}\right) \varphi_{x_{1} x_{1} x_{1}} \leq \Lambda .
\end{aligned}
$$

One obtains from (20)

$$
\varphi_{x_{1} x_{1} x_{1}}=\frac{\varepsilon}{1-\varepsilon \varphi} \varphi_{x_{1}} \varphi_{x_{1} x_{1}}-\psi^{\prime}\left(\varphi_{x_{1}}\right) \varphi_{x_{1} x_{1}}^{2} .
$$

By the Cauchy inequality

$$
\sum_{j \in\{2, \cdots, d\}} \frac{\varphi_{x_{j} x_{j} x_{1}}^{2}}{\varphi_{x_{j} x_{j}}^{2}}+\psi^{\prime}\left(\varphi_{x_{1}}\right)\left[\sum_{i \in\{2, \cdots d\}} \frac{\varphi_{x_{1} x_{i} x_{i}}}{\varphi_{x_{i} x_{i}}}\right] \varphi_{x_{1} x_{1}} \geq-\frac{(d-1)}{4}\left(\psi^{\prime}\left(\varphi_{x_{1}}\right)\right)^{2} \varphi_{x_{1} x_{1}}^{2} .
$$

Hence

$$
\begin{aligned}
& -\frac{d \varepsilon}{1-\varepsilon \varphi} \varphi_{x_{1} x_{1}}-\frac{\varepsilon^{2}}{(1-\varepsilon \varphi)^{2}} \varphi_{x_{1}}^{2}+\frac{\varepsilon}{1-\varepsilon \varphi} \psi^{\prime}\left(\varphi_{x_{1}}\right) \varphi_{x_{1}} \varphi_{x_{1} x_{1}} \\
& +\left[\psi^{\prime \prime}\left(\varphi_{x_{1}}\right)-\frac{d+3}{4}\left(\psi^{\prime}\left(\varphi_{x_{1}}\right)\right)^{2}\right] \varphi_{x_{1} x_{1}}^{2} \leq \Lambda .
\end{aligned}
$$

Applying the Cauchy inequality one gets $\left[\psi^{\prime \prime}-\left(\frac{d+3}{4}+\varepsilon\right)\left(\psi^{\prime}\right)^{2}-d^{2} \varepsilon^{2}\right] \circ \varphi_{x_{1}} \cdot \varphi_{x_{1} x_{1}}^{2}(1-\varepsilon \varphi)^{2} \leq \Lambda(1-\varepsilon \varphi)^{2}+\left(2 \varepsilon^{2} \varphi_{x_{1}}^{2}+\varepsilon\right)$.

Let $\psi_{\varepsilon}$ be a smooth function on $\left[\inf _{y \in K}\left\langle x_{1}, y\right\rangle, \sup _{y \in K}\left\langle x_{1}, y\right\rangle\right]$ satisfying

$$
\psi_{\varepsilon}^{\prime \prime} \geq\left(\frac{d+3}{4}+\varepsilon\right)\left(\psi_{\varepsilon}^{\prime}\right)^{2}+d^{2} \varepsilon^{2}+e^{2 \psi_{\varepsilon}} .
$$


The maximum principle implies

$e^{\psi_{\varepsilon}\left(\varphi_{x_{1}}\right)} \varphi_{x_{1} x_{1}}(1-\varepsilon \varphi)_{+} \leq \max _{x \in \mathbb{R}^{d}}\left(e^{\psi_{\varepsilon}\left(\varphi_{x_{1}}\right)} \varphi_{x_{1} x_{1}}(1-\varepsilon \varphi)_{+}\right) \leq \sqrt{\Lambda+\varepsilon+2 \varepsilon^{2} \sup _{y \in K}\left\langle y, x_{1}\right\rangle^{2}}$.

Set: $f_{\varepsilon}:=e^{-\psi_{\varepsilon}}$. The differential inequality for $\psi_{\varepsilon}$ can be rewritten in the following way:

$$
-f_{\varepsilon} f_{\varepsilon}^{\prime \prime} \geq\left(\frac{d-1}{4}+\varepsilon\right)\left(f_{\varepsilon}^{\prime}\right)^{2}+1+\varepsilon^{2} d^{2} f_{\varepsilon}^{2} .
$$

The following easy-to-check observation completes the proof: there exists a sequence of nonnegative functions $\left\{f_{\varepsilon}\right\}$ on $[-a, a]$ satisfying (24) such that $f_{\varepsilon} \rightarrow$ $f_{\frac{d-1}{4}, a}$ uniformly on $[-a, a]$. In the limit $\varepsilon \rightarrow 0$ we get from (23)

$$
\varphi_{x_{1} x_{1}} \leq \sqrt{\Lambda} f_{\frac{d-1}{4}, a}\left(\varphi_{x_{1}}\right) .
$$

The proof of the first statement is complete.

To prove the second statement we use (17) and (22) to get

$$
\begin{aligned}
& -\frac{d \varepsilon}{1-\varepsilon \varphi} \varphi_{x_{1} x_{1}}-\frac{\varepsilon^{2}}{(1-\varepsilon \varphi)^{2}} \varphi_{x_{1} x_{1}}\left(\frac{\varphi_{x_{1}}^{2}}{\varphi_{x_{1} x_{1}}}+\cdots+\frac{\varphi_{x_{d}}^{2}}{\varphi_{x_{d} x_{d}}}\right)+\sum_{i, j} \frac{\varphi_{x_{i} x_{j} x_{1}}^{2}}{\varphi_{x_{i} x_{i}} \varphi_{x_{j} x_{j}}} \\
& -\sum_{i} \frac{\varphi_{x_{1} x_{1} x_{i}}^{2}}{\varphi_{x_{1} x_{1}} \varphi_{x_{i} x_{i}}}+\psi^{\prime \prime}\left(\varphi_{x_{1}}\right) \varphi_{x_{1} x_{1}}^{2}+\psi^{\prime}\left(\varphi_{x_{1}}\right) V_{x_{1}}(O x) \varphi_{x_{1} x_{1}} \leq \Lambda .
\end{aligned}
$$

Since $\left|V_{x_{i}}\right| \leq M$, arguing as above and passing to the limit $\varepsilon \rightarrow 0$, one gets the desired inequality for $\psi$ :

$$
\psi^{\prime \prime}\left(\varphi_{x_{1}}\right) \varphi_{x_{1} x_{1}}^{2}-M\left|\psi^{\prime}\left(\varphi_{x_{1}}\right)\right| \varphi_{x_{1} x_{1}} \leq \Lambda .
$$

Setting $f=-\log \psi$ and applying Cauchy inequality we obtain that for $f$ satisfying

$$
-f f^{\prime \prime}+\left(1-\frac{\delta}{2} M^{2}\right)\left(f^{\prime}\right)^{2} \geq 1
$$

one has $\varphi_{x_{1} x_{1}} \leq f\left(\varphi_{x_{1}}\right) \sqrt{\Lambda+\frac{1}{2 \delta}}$. Let us set: $-p=1-\frac{\delta}{2} M^{2}$. The proof is complete.

Corollary 4.3. 1) Let $\mu=\gamma$ be the standard Gaussian measure. One has

$$
\left\|D^{2} \varphi\right\| \leq \sqrt{d-1} \frac{\operatorname{diam}(\mathrm{K})}{4 \int_{0}^{\frac{\pi}{2}} \sin ^{\frac{4}{d-1}} x d x} .
$$

2) Let $\mu=\prod_{i=1}^{d} \mu_{i}$, where every $\mu_{i}$ is a copy of a measure $\mu_{0}$ on $\mathbb{R}$ satisfying the following: a) $\mu_{0}=e^{-V} d x$ with smooth $V$, b) $\left|V^{\prime}\right| \leq 1, V^{\prime \prime} \leq 1$. For instance, one can choose $V(x)$ to be equal $|x|$ for large values of $|x|$ and quadratic for small ones. Then

$$
\left\|D^{2} \varphi\right\| \leq \sqrt{-p\left(1+\frac{d}{4(1+p)}\right)} \cdot \frac{\operatorname{diam}(\mathrm{K})}{2 \int_{0}^{\frac{\pi}{2}} \sin ^{-1-\frac{1}{p}} x d x} .
$$

for any $-1<p<0$. 


\section{Appendix : Applications to FUnCtional InEQUALities}

The arguments presented in the following lemma have been communicated to the author by Sasha Sodin. It turns out that convexity condition allows to avoid the use of semigroup techniques in Proposition 2.7 .

Lemma 5.1. For every convex $f$ and unit vector $h$ one has

$$
|\nabla f(x+t h)-f(x)| \leq \frac{2}{t} \sup _{v:|v|=1}(f(x+2 t v)+f(x-2 t v)-2 f(x)) .
$$

Proof. Let us start with $d=1$. Note that

$$
f\left(x_{0}+2 t\right)+f\left(x_{0}-2 t\right)-2 f\left(x_{0}\right)=\int_{-2 t}^{0}\left(f^{\prime}\left(x_{0}+s+2 t\right)-f^{\prime}\left(x_{0}+s\right)\right) d s .
$$

Since $f^{\prime}$ is increasing, one has

$$
f^{\prime}\left(x_{0}+s+2 t\right)-f^{\prime}\left(x_{0}+s\right) \geq f^{\prime}\left(x_{0}+t\right)-f^{\prime}\left(x_{0}\right)
$$

for every $-t \leq s \leq 0$. Thus

$$
\begin{aligned}
f\left(x_{0}+2 t\right)+f\left(x_{0}-2 t\right) & -2 f\left(x_{0}\right) \geq \int_{-t}^{0}\left(f^{\prime}\left(x_{0}+s+2 t\right)-f^{\prime}\left(x_{0}+s\right)\right) d s \\
& \geq \int_{-t}^{0}\left(f^{\prime}\left(x_{0}+t\right)-f^{\prime}\left(x_{0}\right)\right) d s=t\left(f^{\prime}\left(x_{0}+t\right)-f^{\prime}\left(x_{0}\right)\right) .
\end{aligned}
$$

This completes the proof of the one-dimensional case. Let $d \geq 2$. For every couple of unit vectors $u, h$ and $t>0$ one has by the cyclical monotonicity (see 25])

$$
\begin{aligned}
\langle\nabla f(x+t u), t h\rangle+\langle\nabla f(x), t u\rangle+ & \langle\nabla f(x+t h), 0\rangle \\
& \leq\langle\nabla f(x+t h), t h\rangle+\langle\nabla f(x), 0\rangle+\langle\nabla f(x+t u), t u\rangle .
\end{aligned}
$$

Hence

$\langle\nabla f(x+t u)-\nabla f(x), t h\rangle \leq\langle\nabla f(x+t h)-\nabla f(x), t h\rangle+\langle\nabla f(x+t u)-\nabla f(x), t u\rangle$.

Then it follows from the one-dimensional case that

$$
\begin{aligned}
& \langle\nabla f(x+t u)-\nabla f(x), t h\rangle \\
& \leq \frac{1}{t}(f(x+2 t h)+f(x-2 t h)-2 f(x))+\frac{1}{t}(f(x+2 t u)+f(x-2 t u)-2 f(x)) .
\end{aligned}
$$

It remains to take supremum over all unit vectors $h, u$.

This estimate implies generalized continuity of the optimal transport for other types of uniform convexity.

Corollary 5.2. Assume that $W$ satisfies

$$
W(x+y)+W(x-y)-W(x) \geq \delta(|y|)
$$

with some non-negative increasing function $\delta$. Then

$$
|\nabla \varphi(x)-\nabla \varphi(y)| \leq 8 \delta^{-1}\left(4|x-y|^{2}\right) .
$$

Proof. Following the proof of Theorem 2.5. one easily finds that

$$
\varphi(x+t h)+\varphi(x-t h)-2 \varphi(x) \leq 2 t \delta^{-1}\left(t^{2}\right) .
$$

The result follows from Lemma 5.1 
Corollary 5.3. Under assumptions of Corollary $5.2 \nu=e^{-W} d x$ satisfies

$$
\nu\left(A_{r}\right) \geq \Phi\left(\Phi^{-1}(\nu(A))+\frac{1}{2} \sqrt{\delta(r / 8)}\right),
$$

where $\Phi(t)=\frac{1}{\sqrt{2 \pi}} \int_{-\infty}^{t} e^{-\frac{s^{2}}{2}} d s$. In particular, $\nu$ admits a dimension-free concentration property

$$
\nu\left(A_{r}\right) \geq 1-\frac{1}{2} \exp \left(\frac{1}{8} \delta(r / 8)\right)
$$

with $\nu(A) \geq 1 / 2$.

Proof. Consider the optimal mapping $T$ pushing forward the standard Gaussian measure $\gamma=\frac{1}{(2 \pi)^{d / 2}} e^{-\frac{|x|^{2}}{2}} d x$ to $\nu$. The statement follows immediately from Corollary [5.2, the Gaussian isoperimetric inequality (see [6]) and the estimate $\Phi(t) \geq 1-\frac{1}{2} e^{-\frac{t^{2}}{2}}$.

Finally, let us note that similar concentration results can be also derived from the so-called above-tangent lemma. The detailed description, references and various applications can be found in [25], we just mention briefly some important results. The above-tangent estimate have been used by Talagrand 22 for establishing transportation inequality for the standard Gaussian measure. It was understood later that similar arguments can be used for proving a broad class inequalities of Sobolev type. In particular, the Bobkov-Ledoux result has been generalized in [11] (see also [14]). The corresponding isoperimetric inequalities have been proved in [17] by localization arguments. Finally, transportation approach to functional inequalities for non log-concave measures has been developed in [3].

We note that there exists another measure of convexity, which is especially convenient when one deals with the above-tangent arguments.

Remark 5.4. Everywhere below we deal with an arbitrary (non-Euclidean) norm $\|\cdot\|$ on $\mathbb{R}^{d}$.

For a convex $W$ and $\|\cdot\|$ let us define $\delta: \mathbb{R}^{+} \rightarrow \mathbb{R}^{+}$and $b: \mathbb{R}^{+} \rightarrow \mathbb{R}^{+}$in the following way:

$$
\begin{aligned}
& \delta(t)=\inf \{W(x+y)+W(x-y)-2 W(x):\|y\| \geq t\} \\
& b(t)=\inf \{W(x+y)-W(x)-\langle\nabla W(x), y\rangle:\|y\| \geq t\}
\end{aligned}
$$

These quantities are equivalent in a sense.

Lemma 5.5. One has

$$
b(2 t)-2 b(t) \geq \delta(t) \geq 2 b(t)
$$

Proof. Relation $\delta(t) \geq 2 b(t)$ follows from

$$
\begin{aligned}
& W(x+y)+W(x-y)-2 W(x)= \\
& \quad(W(x+y)-W(x)-\langle\nabla W(x), y\rangle)+(W(x-y)-W(x)-\langle\nabla W(x),-y\rangle) .
\end{aligned}
$$

Further, note that

$$
W\left(x+\frac{y}{2}\right)-W(x)-\left\langle\nabla W(x), \frac{y}{2}\right\rangle \geq b(t / 2) .
$$


Taking $y$ with $|y|=t$ one has

$\delta(t / 2) \leq W(x+y)+W(x)-2 W\left(x+\frac{y}{2}\right) \leq W(x+y)-W(x)-\langle\nabla W(x), y\rangle-2 b(t / 2)$.

This clearly implies $\delta(t / 2)+2 b(t / 2) \leq b(t)$.

Definition 5.6. In what follows let

1) $\tilde{b}$ be the maximal convex function majorized by $b$

2) $b^{*}$ be the corresponding convex conjugated function:

$$
b^{*}(t)=\sup _{s>0}\{t s-b(s)\} .
$$

The results of the following proposition are known (see [11]). For the reader convenience we just sketch the proof of 2).

Proposition 5.7. Let $W$ be a convex function such that $\nu=e^{-W} d x$ is a probability measure. Consider a norm $\|\cdot\|$ on $\mathbb{R}^{d}$ and define $b$ by (25). Let $f \cdot \nu$ be a probability measure and $T=\nabla \varphi$ be the optimal transportation mapping sending $\nu$ to $f \cdot \nu$. Then the following holds:

1) Talagrand's type inequality:

$$
\int_{\mathbb{R}^{d}} b(\|\nabla \varphi(x)-x\|) d \nu \leq \int_{\mathbb{R}^{d}} f \log f d \nu
$$

2) a modified log-Sobolev-type inequality

$$
\int_{\mathbb{R}^{d}} f \log f d \nu \leq \int_{\mathbb{R}^{d}} b^{*}\left(\left\|\frac{\nabla f(x)}{f(x)}\right\|_{*}\right) f d \nu,
$$

where $\|\cdot\|_{*}$ is the corresponding dual norm

Proof. Let as use the above-tangent arguments. Let $T=\nabla \varphi$ be the optimal transportation sending $f \cdot \nu$ to $\nu$. By the change of variables formula

$$
0=W(x)-W(\nabla \varphi)+\log \operatorname{det} D_{a}^{2} \varphi-\log f(x),
$$

where $D_{a}^{2} \varphi$ is the second Alexandroff derivative of $\varphi$ (see [25] for details). Hence

$$
\begin{aligned}
\log f= & W(x)-W(\nabla \varphi)+\log \operatorname{det} D_{a}^{2} \varphi= \\
& W(x)-W(\nabla \varphi)-\langle\nabla W(x), x-\nabla \varphi\rangle \\
& +\langle\nabla W(x), x-\nabla \varphi\rangle+\log \operatorname{det} D_{a}^{2} \varphi \\
& \leq-b(\|\nabla \varphi(x)-x\|)+\langle\nabla W(x), x-\nabla \varphi\rangle+\log \operatorname{det} D_{a}^{2} \varphi .
\end{aligned}
$$

Let us integrate this formula with respect to $f \cdot \nu$. Integration by parts and convexity properties of $\varphi$ give

$$
\begin{aligned}
\int_{\mathbb{R}^{d}} f \log f d \nu & \leq-\int_{\mathbb{R}^{d}} b(\|\nabla \varphi(x)-x\|) f d \nu+\int_{\mathbb{R}^{d}}\langle\nabla f(x), x-\nabla \varphi\rangle d \nu . \\
& -\int_{\mathbb{R}^{d}}\left(\operatorname{Tr} D_{a}^{2} \varphi-d-\log \operatorname{det} D_{a}^{2} \varphi\right) d \mu .
\end{aligned}
$$

It is well-known and easy to verify that $\operatorname{Tr} D_{a}^{2} \varphi-d-\log \operatorname{det} D_{a}^{2} \varphi \geq 0$. Applying

$$
\left\langle\frac{\nabla f(x)}{f(x)}, x-\nabla \varphi\right\rangle \leq\|x-\varphi(x)\| \cdot\left\|\frac{\nabla f(x)}{f(x)}\right\|_{*}
$$

and the Young inequality one completes the proof. 
Finally, the following concentration inequality

$$
\begin{gathered}
\nu\left(A_{r}\right) \geq 1-2 e^{-2 \tilde{b}(r / 2)}, \\
\nu(A) \geq \frac{1}{2}, A_{r}=\{y:\|x-y\| \geq r\}
\end{gathered}
$$

can be easily verified by a modification of Marton's arguments. Let $T_{1}=\nabla \varphi_{1}$, $T_{2}=\nabla \varphi_{2}$ be a couple of optimal transportation mappling pushing forward $\nu$ to $\nu_{1}=\left.\frac{1}{\nu(A)} \nu\right|_{A}$ and $\nu_{2}=\left.\frac{1}{\nu\left(A_{r}^{c}\right)} \nu\right|_{A_{r}^{c}}$ respectively. Then $T=T_{2} \circ T_{1}^{-1}$ sends $\nu_{1}$ to $\nu_{2}$. One has

$$
\tilde{b}(r / 2) \leq \int_{\mathbb{R}^{d}} \tilde{b}\left(\frac{1}{2}\|x-T(x)\|\right) d \nu_{1}=\int_{\mathbb{R}^{d}} \tilde{b}\left(\frac{1}{2}\left\|T_{1}-T_{2}\right\|\right) d \nu .
$$

Hence by convexity of $\tilde{b}$ and the Talagrand's type estimate

$$
\begin{aligned}
2 \tilde{b}(r / 2) & \leq \int_{\mathbb{R}^{d}} \tilde{b}\left(\left\|T_{1}(x)-x\right\|\right) d \nu+\int_{\mathbb{R}^{d}} \tilde{b}\left(\left\|T_{2}(x)-x\right\|\right) d \nu \\
& \leq \log \left(\frac{1}{\nu(A)}\right)+\log \left(\frac{1}{\nu\left(A_{r}^{c}\right)}\right) .
\end{aligned}
$$

Hence

$$
e^{2 \tilde{b}(r)} \leq \frac{1}{\nu(A) \nu\left(A_{r}^{c}\right)}
$$

This work was supported by the RFBR project 07-01-00536, GFEN-06-01-39003, RF President Grant MD-764.2008.1, DFG Grant 436 RUS 113/343/0(R).

\section{REFERENCES}

[1] Bakry D., Emery M., "Diffusions hypercontractives", In Séminaire de Probabilités XIX, Lect. Notes in Math., Springer 1123 (1985), 179-206.

[2] Bakry D., Ledoux M., "Lévy-Gromov's isoperimetric inequality for an infinite-dimensional diffusion generator", Invent. Math. 123 (1996), 259-281.

[3] Barthe F., Kolesnikov A.V., "Mass transport and variants of the logarithmic Sobolev inequality", Journal. Geom. Analysis 18:4, (2008), 921-979 (arXiv:0709.3890).

[4] Bobkov S., Houdre C., "Isoperimetric constants for product probability measures", Ann. Probab. 25, (1997), 184-205.

[5] Bobkov S., Ledoux M., "From Brunn-Minkowski to Brascamp-Lieb and to logarithmic Sobolev inequalities", Geom. Funct. Anal. 10:5, (2008), 1028-1052.

[6] Bogachev V.I., Gaussian measures, Amer. Math. Soc., Providence, Rhode Island, 1998.

[7] Caffarelli L.A., "The regularity of mappings with a convex potential", Journal of Amer. Math. Soc. 5: 1, (1992), 99-104.

[8] Caffarelli L.A., "Boundary regularity of maps with a convex potentials. II", Ann. of Math.(2), 144: 3, (1996), 453-496.

[9] Caffarelli L.A., "Monotonicity properties of optimal transportation and the FKG and related inequalities", Comm. Math. Phys. 214:3, (2000), 547-563.

[10] Caffarelli L.A., Cabré X., Fully nonlinear elliptic equations, Amer. Math. Soc., Providence, RI, 1995.

[11] Cordero-Erausquin D., Gangbo W., Houdré C., "Inequalities for generalized entropies and optimal transportation", Recent advances in the theory and applications of mass transport. - Contemp. Math. Amer. Math. Soc., 353, Providence, RI, (2004), 73-94.

[12] Gutièrrez C.E., The Monge-Ampère equation, Progress in Nonlinear Differential Equations and Their Applications, 44, Birkhäuser, 2001.

[13] Hargé G., " A convex/log-concave correlation inequality for Gaussian measure and an application to abstract Wiener spaces", Probab. Theory Related Fields, 13:3, (2004), 415-440.

[14] Kolesnikov A.V., "Convexity inequalities and optimal transport of infinite-dimensional measures", J. Math. Pures Appl., 83:11, (2004), 1373-1404. 
[15] Krylov N.V., " Fully nonlinear second order elliptic equations: recent developments", Ann. Scuola Norm. Sup. Pisa Cl. Sci., 4, Vol. XXV (1997), 569-595.

[16] Ledoux M. The concentration of measure phenomenon, Mathematical Surveys and Monographs 89. Amer. Math. Soc., 2001.

[17] Milman E., Sodin S., "An isoperimetric inequality for uniformly log-concave measures and uniformly convex bodies", Jour. Funct. Anal., 254:5, (2008), 1235-1268. (arXiv:0703857).

[18] Payne L. E, Weinberger H.F., "An optimal Poincaré inequality for convex domains", Arch. Rational Mech. Anal., 5, (1960), 286-292.

[19] Pogorelov A.V., "On the regularity of generalized solutions of the equation $\operatorname{det}\left(\frac{\partial^{2} u}{\partial x^{i} \partial x^{j}}\right)=$ $\phi\left(x^{1}, \cdots, x^{n}\right)>0 "$, Soviet Math. Dokl., 12: 5, (1971), 1436-1440.

[20] Stein E.M., Singular integrals and differentiability properties of functions, Princeton Univ. Press, Prinston, New Jersey, 1970.

[21] Talagrand M., "A new isoperimetric inequalities for product measure, and the concentration of measure phenomenon", GAFA (Israel Seminar, 1989-1990). Lecture Notes in Math. 1469, (1991), 91-124, Springer.

[22] Talagrand M., "Transportation cost for Gaussian and other product measures", Geom. Funct. Anal., 6, (1996), 587-600.

[23] Urbas J., Mass transfer problems, Lecture Notes, Univ. of Bonn, 1998.

[24] Valdimarsson S.I., "On the Hessian of the optimal transport potential" (preprint).

[25] Villani C., Topics in Optimal Transportation, Amer. Math. Soc. Providence, Rhode Island, 2003. 